\title{
The myth of recovery from mental illness
}

\author{
David Whitwell
}

Recovery from mental lllness is a fairly straightforward concept to members of the general public: "am I going to recover", "what are the chances of recovery", or "is our son/daughter ever likely to recover"? These are the sorts of questions that doctors and psychiatrists get asked every day.

Recovery is generally taken to mean getting back to how you were before the illness started, being restored to your former state. It implies that you can do the same things that you could before you were ill and that you will feel the same. It involves being the same as before. This conception of recovery, is of recovery as a state of a person, as the end state following a period of illness. It is the goal which is very much sought.

There is a tendency to answer questions about recovery by saying "yes, of course you will recover". It may be qualified by "it may take a long time" or "we can't really give a definite answer". Doctors in the past were notorious for giving vague evastve answers, 'soft soap' and platitudes. Doctors are caught in a bind of being asked to make the patient better, to improve their morale and at the same time provide information. In the past attempts to raise morale was seen as more important than providing accurate information.

There is now a real move towards sharing information with patients and involving them in the decision-making process. So, when we are asked about recovery we should try to give accurate information. The problem is that this is in short supply.

The question of whether a person will recover from mental illness (in the sense understood by the general public) is an empirical one. In any individual case it will always be uncertain, although in time the answer will become clear. As well as questions about the overall likelihood of recovery there are many other questions. What does recovery feel like? How do people recognise it? What may hasten or hinder it?

Research studies use rating scales which score large numbers of symptoms. Statistically significant reductions in scores are sought. The language of research studies does not convey answers to questions about recovery. A qualitative study was undertaken to try to learn more about recovery. The strategy was to identify a group of people who had recovered from mental illness and by interviewing them learn about the process and the experience of recovery.

A pilot study was undertaken to try out the interview. Subjects for the pilot study were volunteers from a users support group for expsychiatric patients. The interviews went well and information about the experience of recovery seemed to be readily available. The volunteers welcomed the involvement in the project. Ten volunteers were interviewed. The findings of the pilot study immediately put the future of the project in doubt. Although the volunteers had come forward identifying themselves as people who had recovered, their accounts made this look quite uncertain. They reported major continuing problems due to their psychiatric illness. These included continuing symptoms, reduced tolerance of stress, restricted lifestyle, unemployment, stigma and poverty. They made remarks such as:

'Yes, but I will never be the same person again".

"I have made a good recovery, but I wouldn't say I have recovered. As to making a full recovery, I don't know if you ever do".

"I do consider that I have recovered, but I think it will take a little bit longer to forget".

The volunteers tended to see themselves as survivors. From their accounts it was clear why they preferred that term. None of them could definitely see themselves as having recovered.

The next stage of the study was to interview a series of patients who were identified by their psychiatrists as 'having recovered'. At the outset the psychiatrists saw no difficulty in finding suitable subjects. These were drawn from all the patients being treated in a busy psychiatric unit serving a 240000 population. In practice subjects were very hard to find. Over nine months only 13 people were identified, too few for any firm conclusions. However, the failure to identify a sizeable group of people who had 'recovered from psychiatric illness', and the 
observations of the few who were found are of interest.

Almost all the subjects took issue with the concept of recovery. They all wanted to say that they had 'not really recovered'. They were strongly aware of not being the same as they had been. They were more careful, less confident, more aware of danger. A few saw this change in a positive light, saying that they were now more genuine, more their real self, more sensitive to other people. However, the feeling of "not being the same" was felt to be inconsistent with the concept of recovery. The subjects were also highly conscious of their impaired life position following their psychiatric illness. They described unemployment, divorce, housing problems, lack of money and social isolation. Subjects also described continuing psychological sequelae of their illness including reduced tolerance of stress, loss of self-confidence and recurring unpleasant memories.

This small study of routine National Health Service (NHS) practice suggests that it is not common for patients being treated to end up in a state that they identify as one of "having recovered'. This does not mean that recovery as described does not occur. However, it does mean that in standard practice it is very far from being the norm. This may be particularly true for serious mental illness which is now the main focus of NHS psychiatry.

\section{Why does this matter?}

Recovery is part of common discourse. When people are told that they can expect to recover if they follow certain advice, they will expect to recover. If doctors and nurses believe that satisfactory treatment will result in recovery they will review the great majority of their cases as less than satisfactory.

In this way recovery can act as a mirage leading on towards something which is not really there. Part of the confusion arises from the way the concept is used. People tend to understand recovery as an end state which will be achieved. This is what is misleading. Recovery is also a process which people become engaged in and along which they make more or less progress.

Recovery is a concept which is left over from an over simplified medical model of psychiatric illness. Psychiatrists like to think that they have moved beyond simple medical models but. insofar as they continue to focus on the treatment of episodes of illness as the main thrust for intervention, they remain firmly attached to that model.

Some patients, particularly those who belong to a user group, apply the term 'survivor' to themselves. The term is generally unpopular with psychiatrists because of its negative implications. Such people often describe themselves as survivors of psychiatry as much as of mental illness. However, it may be an empirical truth that surviving mental illness is a better description than recovery. Surviving the damage and coping with disability and disadvantage are alternative models to the illness model.

These considerations may seem excessively negative and pessimistic. Surely it is better to hope for the best even if it is rarely achieved? However, there is a much more positive side to all this. The hope to be how you were before, to be unchanged and unscathed after terrible experiences is probably very primitive. It is a type of denial. It is the hope that we will not be harmed in life, will not be damaged and grow old and die. It is understandable and widespread, but is not reality.

It is supported by over optimistic therapists of all varieties. More and more complex and specific psychological therapies can seem to offer the promise of undoing the harm done by the world. Reality is that people are battered and altered by experience. The self is damaged. However, this damage to the self is not all bad. The strength to carry on and survive personal damage is one of the perennial themes of art and literature. Teachers, healers and charismatic leaders do not present themselves as fresh and untouched by the world. They derive strength from the damage that they have sustained and overcome. This seems to be insufficiently recognised in psychological theories which continue to offer cures to all ills.

\section{Acknowledgements}

I would like to thank Ms Bree McDonald for conducting the interviews, my colleagues at Southmead Hospital for their encouragement and support, members of Southmead Users Network for their cooperation and the Southmead Hospital Research Foundation for financial support.

David Whitwell, Consultant Psychiatrist, Southmead Hospital, Westbury on Trym. Bristol BS10 5NB 